\title{
Colouring the Square of the Cartesian Product of Trees
}

\author{
David R. Wood $\|^{\dagger}$ \\ Department of Mathematics and Statistics, The University of Melbourne, Melbourne, Australia \\ received $16^{\text {th }}$ August 2010, accepted $4^{\text {th }}$ August 2011.
}

We prove upper and lower bounds on the chromatic number of the square of the cartesian product of trees. The bounds are equal if each tree has even maximum degree.

Keywords: cartesian product, colouring, square graph

\section{Introduction}

This paper studies colourings of the square of cartesian products of trees. For simplicity we assume that a tree has at least one edge.

For our purposes, a colouring of a graph $G$ is a function $c: V(G) \rightarrow \mathbb{Z}$ such that $c(v) \neq c(w)$ for every edge $v w$ of $G$. The square graph $G^{2}$ of $G$ has vertex set $V(G)$, where two vertices are adjacent in $G^{2}$ whenever they are adjacent in $G$ or have a common neighbour in $G$. Thus, a colouring of $G^{2}$ corresponds to a colouring of $G$, such that in addition, vertices with a common neighbour in $G$ are assigned distinct colours.

Let $[a, b]:=\{a, a+1, \ldots, b\}$. The cartesian product of graphs $G_{1}, \ldots, G_{d}$ is the graph $G_{1} \square \cdots \square G_{d}$ with vertex set $\left\{\left(v_{1}, \ldots, v_{d}\right): v_{i} \in V\left(G_{i}\right)\right\}$, where vertices $v=\left(v_{1}, \ldots, v_{d}\right)$ and $w=\left(w_{1}, \ldots, w_{d}\right)$ are adjacent whenever $v_{i} w_{i} \in E\left(G_{i}\right)$ for some $i \in[1, d]$, and $v_{j}=w_{j}$ for all $j \neq i$. In this case, $v w$ is in dimension $i$. Let $\Delta(G)$ be the maximum degree of $G$.

Theorem 1 Let $T_{1}, \ldots, T_{d}$ be trees. Let $G:=T_{1} \square T_{2} \square \cdots \square T_{d}$. Then

$$
1+\sum_{i=1}^{d} \Delta\left(T_{i}\right) \leq \chi\left(G^{2}\right) \leq 1+2 \sum_{i=1}^{d}\left\lceil\frac{1}{2} \Delta\left(T_{i}\right)\right\rceil .
$$

This upper bound improves upon a similar bound by Jamison et al. (2006), who proved $\chi\left(G^{2}\right) \leq$ $1+2 \sum_{i=1}^{d}\left(\Delta\left(T_{i}\right)-1\right)$, assuming that each $\Delta\left(T_{i}\right) \geq 2$. Theorem 1 implies:

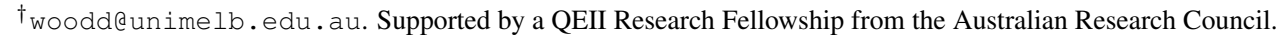


Corollary 1 Let $T_{1}, \ldots, T_{d}$ be trees, such that $\Delta\left(T_{i}\right)$ is even for all $i \in[1, d]$. Let $G:=T_{1} \square T_{2} \square \cdots \square T_{d}$. Then

$$
\chi\left(G^{2}\right)=1+\sum_{i=1}^{d} \Delta\left(T_{i}\right) .
$$

This corollary generalises a result of Fertin et al. (2003), who proved it when each $T_{i}$ is a path, and thus $G$ is a $d$-dimensional grid. See (Sopena and Wu, 2010, Fertin et al., 2004, Pór and Wood, 2009, Jamison et al., 2006, Chiang and Yan, 2008) for more related results.

\section{The Proof}

For a colouring $c$ of a graph $G$, the span of an edge $v w$ of $G$ is $|c(v)-c(w)|$. The following lemma is well known; see (Pór and Wood, 2009) for example.

Lemma 1 Let $G$ be a graph. If $G^{2}$ has a colouring in which every edge of $G$ has span at most $s$, then $G^{2}$ is $(2 s+1)$-colourable.

Proof: Let $c: V\left(G^{2}\right) \rightarrow \mathbb{Z}$ be the given colouring of $G^{2}$. Since every edge of $G$ has span at most $s$, every edge of $G^{2}$ has span at most $2 s$. Let $c^{\prime}(v):=c(v) \bmod (2 s+1)$ for each vertex $v$. Then $c^{\prime}(v) \neq c^{\prime}(w)$ for each edge $v w$ of $G^{2}$. Thus $G^{2}$ is $(2 s+1)$-colourable.

Lemma 2 For every tree $T$ and non-negative integer $s, T^{2}$ has a colouring such that every edge of $T$ has span in $\left[s+1, s+\left\lceil\frac{1}{2} \Delta(T)\right\rceil\right]$.

Proof: We proceed by induction on $|V(T)|$. If $|V(T)|=2$ the result is trivial. Now assume that $|V(T)| \geq 3$. Let $v$ be a leaf vertex of $T$. Let $w$ be the neighbour of $v$. By induction, $(T-v)^{2}$ has a colouring $c$ such that every edge of $T-v$ has span in $\left[s+1, s+\left\lceil\frac{1}{2} \Delta(T)\right\rceil\right]$. Let

$$
X:=\left\{x \in \mathbb{Z}:|x| \in\left[s+1, s+\left\lceil\frac{1}{2} \Delta(T)\right]\right]\right\} .
$$

Each neighbour of $w$ in $T-v$ is coloured $c(w)+x$ for some $x \in X$. Since $|X| \geq \Delta(T)$ and $w$ has degree less than $\Delta(T)$ in $T-v$, for some $x \in X$, no neighbour of $w$ is coloured $c(w)+x$. Set $c(v):=c(w)+x$. Thus $|c(v)-c(w)|=|x| \in\left[s+1, s+\left\lceil\frac{1}{2} \Delta(T)\right]\right]$. No two neighbours of $w$ receive the same colour. Hence $c$ is the desired colouring of $T$.

Proof of Theorem 1: The lower bound is well known (Jamison et al. 2006). In particular, for $i \in[1, d]$, let $v_{i}$ be a vertex of maximum degree in $T_{i}$. Then $\left(v_{1}, \ldots, v_{d}\right)$ has degree $\sum_{i} \Delta\left(T_{i}\right)$ in $G$. This vertex and its neighbours in $G$ receive distinct colours in any colouring of $G^{2}$. Thus $\chi\left(G^{2}\right) \geq 1+\sum_{i} \Delta\left(T_{i}\right)$.

Now we prove the upper bound. Let $s_{1}:=0$ and $s_{i}:=\sum_{j=1}^{i-1}\left\lceil\frac{1}{2} \Delta\left(T_{j}\right)\right\rceil$. By Lemma $2, T_{i}^{2}$ has a colouring $c_{i}$ such that every edge of $T_{i}$ has span in $\left[s_{i}+1, s_{i}+\left\lceil\frac{1}{2} \Delta\left(T_{i}\right)\right\rceil\right]$. Thus the spans of edges in distinct trees are distinct.

Colour each vertex $v=\left(v_{1}, \ldots, v_{d}\right)$ of $G$ by $c(v):=\sum_{i=1}^{d} c_{i}\left(v_{i}\right)$.

Suppose on the contrary that $c(v)=c(w)$ for some edge $v w$ of $G$. Say $v w$ is in dimension $i$. Thus $v_{j}=w_{j}$ for all $j \neq i$. Hence $c_{i}\left(v_{i}\right)=c_{i}\left(w_{i}\right)$, and $c_{i}$ is not a colouring of $G$. This contradiction proves that $c$ is a colouring of $G$. 
Suppose on the contrary that $c(x)=c(y)$ for two vertices $x$ and $y$ with a common neighbour $v$ in $G$. Say $v x$ is in dimension $i$, and $v y$ is in dimension $j$. Thus $v_{\ell}=x_{\ell}$ for all $\ell \neq i$, and $v_{\ell}=y_{\ell}$ for all $\ell \neq j$. Now $c_{i}\left(x_{i}\right)-c_{i}\left(v_{i}\right)=c(x)-c(v)=c(y)-c(v)=c_{j}\left(y_{j}\right)-c_{j}\left(v_{j}\right)$. Thus the edges $x_{i} v_{i}$ and $y_{j} v_{j}$ have the same span. Since the spans of edges in distinct trees are distinct, $i=j$. Hence $c_{i}\left(x_{i}\right)=c_{i}\left(y_{i}\right)$. However, $v_{i}$ is a common neighbour of $x_{i}$ and $y_{i}$ in $T_{i}$, implying $c_{i}$ is not a colouring of $T_{i}^{2}$. This contradiction proves that $c$ is a colouring of $G^{2}$.

Each edge of $G$ has span at most $\sum_{i=1}^{d}\left\lceil\frac{1}{2} \Delta\left(T_{i}\right)\right\rceil$. The result follows from Lemma 1

\section{References}

S.-H. Chiang and J.-H. Yan. On $L(d, 1)$-labeling of Cartesian product of a cycle and a path. Discrete Appl. Math., 156(15):2867-2881, 2008. doi:10.1016/j.dam.2007.11.019.

G. Fertin, E. Godard, and A. Raspaud. Acyclic and k-distance coloring of the grid. Inform. Process. Lett., 87(1):51-58, 2003. doi:10.1016/S0020-0190(03)00232-1

G. Fertin, A. Raspaud, and B. Reed. Star coloring of graphs. J. Graph Theory, 47(3):163-182, 2004. doi:10.1002/jgt.20029.

R. E. Jamison, G. L. Matthews, and J. Villalpando. Acyclic colorings of products of trees. Inform. Process. Lett., 99(1):7-12, 2006. doi:10.1016/j.ipl.2005.11.023

A. Pór and D. R. Wood. Colourings of the Cartesian product of graphs and multiplicative Sidon sets. Combinatorica, 29(4):449-466, 2009. doi:10.1007/s00493-009-2257-0

E. Sopena and J. Wu. Coloring the square of the cartesian product of two cycles. Discrete Math., 310 (17-18):2327-2333, 2010. doi:10.1016/j.disc.2010.05.011. 
